\title{
Flexible distribution functions, higher-order preferences and optimal portfolio allocation
}

\author{
Trino-Manuel Ñ́guez \\ Department of Economics and Quantitative Methods, Westminster Business School, \\ University of Westminster, London NW1 5LS, UK \\ Ivan Paya* \\ Department of Economics, Lancaster University Management School, Lancaster LA1 4YX, UK \\ David Peel \\ Department of Economics, Lancaster University Management School, Lancaster LA1 4YX, UK \\ Javier Perote \\ Department of Economics, University of Salamanca, Salamanca 37007, Spain
}

\begin{abstract}
In this paper we show that flexible probability distribution functions, in addition to been able to capture stylized facts of financial returns, can be used to identify pure higher-order effects of investors' optimizing behavior. We employ the five-parameter weighted generalized beta of the second kind distribution -and other density functions nested within it- to determine the conditions under which risk averse, prudent and temperate agents are diversifiers in the standard portfolio choice theory. Within this framework, we illustrate through comparative statics the economic significance of higher-order moments in return's distributions.
\end{abstract}

Keywords: Decision analysis; Higher-order moments and preferences; Portfolio choice; Weighted generalized beta of the second kind.

JEL classification: D81, G11.

*Corresponding author: Tel: +44 1524593 504; fax: +44 1524594 244; email: i.paya@lancaster.ac.uk. 


\section{Introduction}

Flexible probability distribution functions (pdfs), which we refer to as pdfs with more than two parameters, are typically employed in the finance literature to capture asset returns skewness and excess kurtosis. Economic theory shows that higher moments of distribution matter for asset allocation because they are closely linked to higher-order risk preferences such as downside risk aversion or prudence, and temperance (see Menezes et al., 1980; Kimball, 1990; Guidolin and Timmermann, 2008).

In this context we show that the five-parameter weighted generalized beta of the second kind (WGB2) pdf is of particular interest because it allows to identify the relationship between higher-order moments and 'pure' higher-order risk preferences. The WGB2 nests densities such as the generalized beta of the second kind (GB2), generalized gamma (GG), log-normal (LN), gamma (g) and Weibull (W), which is critical to isolate the direct relationship between $\mathrm{n}$ th-order moments and $\mathrm{n}$ th-order risk attitudes. ${ }^{1}$

The flexibility of the WGB2 is clearly an advantage relative to two-parameter pdfs that have limitations in portfolio choice analysis. This is the case because pdfs can only be comparable in terms of one risk preference but not in terms of more than one. To illustrate our argument we employ the example given by Feldstein (1969) in his seminal paper. Feldstein demonstrated that the investor's decision to do not diversify, or plunge, i.e., optimally allocate all their wealth in risky assets, could occur for reasonable values of expected return and variance of the risky asset assuming log-utility and a log-normal distribution. $^{2}$ We show that flexible pdfs can keep low(er) moments fixed while modifying others, high(er) moments, that capture risk preferences other than risk aversion. We derive the conditions, within the standard portfolio choice model, under which investors that are risk averse, prudent and temperate, are diversifiers. Our analysis thus critically illustrates the role of the assumed pdf on the relation of higher moments and portfolio decision within this simple, yet relevant, theoretical framework.

\section{Theoretical framework: portfolio choice model}

Consider a two-asset (risky/riskless) economy in which an investor with initial wealth $\omega_{0}$ decides to invest a proportion, $0 \leq \theta \leq 1$ (ruling out short selling), in the risky asset so that

\footnotetext{
${ }^{1}$ See McDonald (1984) and Ye et al. (2012) for the theoretical properties of these densities and their applications in economics and finance.

${ }^{2}$ Agents investing all their liquid wealth in risky assets is a common assumption in models of asset allocation (see e.g. Brunnermeier and Nagel, 2008).
} 
after one period expected wealth becomes

$$
\bar{\omega}=(1-\theta) \omega_{0}+\theta \omega_{0} E(r)
$$

where $E(r)$ is the expected gross rate of return of the risky asset.

For the investor's preferences we assume a $\log$-utility function, $u_{1}(\omega)=\ln (\omega){ }^{3}$ This utility displays the features that characterize higher-order risk preferences such as prudence (or downside risk) and temperance, i.e. $u^{\prime \prime \prime}>0$, and $u^{i v}<0$, respectively (Eeckhoudt and Schlesinger, 2006). ${ }^{4}$

The representative agent maximizes her expected utility (EU) by choosing the proportion $\theta$ to invest in the risky asset,

$$
\begin{aligned}
\max _{\{\theta\}} E_{f}[u(\omega)] & =\max _{\{\theta\}} E_{f}\left[u\left((1-\theta) \omega_{0}+\theta \omega_{0} r\right)\right] \\
& =\max _{\{\theta\}} E_{f}\left\{u\left(\omega_{0}[1+\theta(r-1)]\right)\right\} .
\end{aligned}
$$

Following the analysis in Feldstein, the conditions under which the investor will maximize $E_{f}[u(\omega)]$ by holding only the risky asset are,

$$
\begin{aligned}
\xi_{f}(u(\omega) ; \theta) & >0 \forall \theta \in[0,1) \\
\left.\xi_{f}(u(\omega) ; \theta)\right|_{\theta=1} & \geq 0
\end{aligned}
$$

where $\xi_{f}(u(\omega) ; \theta) \equiv \frac{\partial E_{f}[u(\omega)]}{\partial \theta}$. For log-utility (2) becomes $^{5}$

$$
\max _{\{\theta\}}\left(E_{f}\left[u_{1}(\omega)\right]\right)=\max _{\{\theta\}}\left(\ln \left(\omega_{0}\right)+E_{f}\{\ln [1+\theta(r-1)]\}\right) .
$$

$\xi_{f}\left(u_{1}(\omega) ; \theta\right) \equiv E_{f}\left[\frac{r-1}{1+\theta(r-1)}\right]$ is (i) positive for $\theta=0$ (equation (6)), and (ii) a strictly decreasing function of $\theta$ for all $\theta \in[0,1]$ (equation (7)), ${ }^{6}$ therefore $E_{f}\left[u_{1}(\omega)\right]$ has a unique global maximum at $\theta=1$ for all admissible $\theta$ if condition $\left.(8) \xi_{f}\left(u_{1}(\omega) ; \theta\right)\right|_{\theta=1} \geq 0$ holds

\footnotetext{
${ }^{3}$ For example Gandelman and Hernández-Murillo (2014) estimate the coefficient of relative risk aversion for 75 countries and suggest that it varies closely around one. Hartley et al. (2014), in the context of attitudes to risk in a game show, suggest that log-utility is a good approximation to agents' utility function.

${ }^{4}$ We extended the analysis by considering an alternative (power) utility function for which we obtained necessary (but not sufficient) plunging conditions. These results are available from the authors upon request.

${ }^{5}$ This model could easily be extended to a dynamic framework because for log-utility the optimal oneperiod $\theta$ is the same as the multi-period $\theta$ (Brandt, 2010).

${ }^{6}$ Note that $\frac{\partial E_{f}[u(\omega)]}{\partial \theta}=\frac{\partial}{\partial \theta} \int u(\omega) f(r) d r=\int \frac{\partial u(\omega)}{\partial \theta} f(r) d r=E_{f}\left[\frac{\partial u(\omega)}{\partial \theta}\right]$.
} 
(Feldstein, 1969, p.9),

$$
\begin{aligned}
\left.\xi_{f}\left(u_{1}(\omega) ; \theta\right)\right|_{\theta=0} & =E_{f}[r-1]>0, \\
\frac{\partial \xi_{f}\left(u_{1}(\omega) ; \theta\right)}{\partial \theta} & =-E_{f}\left[\frac{(r-1)^{2}}{(1+\theta(r-1))^{2}}\right]<0 \forall \theta \in[0,1], \\
\left.\xi_{f}\left(u_{1}(\omega) ; \theta\right)\right|_{\theta=1} & =1-E_{f}\left(r^{-1}\right) \geq 0 .
\end{aligned}
$$

\subsection{Conditions for optimal choice under alternative return's pdfs}

In this section we derive the theoretical condition for optimal choice (8) under alternative return's pdfs and express it in terms of the pdfs' central and raw moments. Table 1 displays the density and moment generating functions (mgfs) for the five-, four- and three-parameter pdfs WGB2, GB2, and GG, respectively.

\section{[Insert Table 1]}

For the WGB2 case, using its mgf and the expression for $b$ derived from its first raw moment, we can express condition (8) as,

$$
\begin{aligned}
1-E_{f}\left(r^{-1}\right) & =1-\frac{1}{b} \frac{\Gamma\left(p+\frac{k}{c}-\frac{1}{c}\right) \Gamma\left(q-\frac{k}{c}+\frac{1}{c}\right)}{\Gamma\left(p+\frac{k}{c}\right) \Gamma\left(q-\frac{k}{c}\right)} \geq 0 \\
m_{1, W G B 2} & \geq \frac{\Gamma\left(p+\frac{k}{c}+\frac{1}{c}\right) \Gamma\left(q-\frac{k}{c}-\frac{1}{c}\right) \Gamma\left(p+\frac{k}{c}-\frac{1}{c}\right) \Gamma\left(q-\frac{k}{c}+\frac{1}{c}\right)}{\Gamma^{2}\left(p+\frac{k}{c}\right) \Gamma^{2}\left(q-\frac{k}{c}\right)} .
\end{aligned}
$$

Hereafter $m_{t, f}$ denotes the th-central moment of $f$. Similarly, for the GB2 pdf the optimal condition is

$$
m_{1, G B 2} \geq \frac{\Gamma\left(p+\frac{1}{c}\right) \Gamma\left(q-\frac{1}{c}\right) \Gamma\left(p-\frac{1}{c}\right) \Gamma\left(q+\frac{1}{c}\right)}{\Gamma(p)^{2} \Gamma(q)^{2}} .
$$

McDonald (1984) demonstrates that the substitution $b=q^{1 / c} / a$ as $q \rightarrow \infty$ in the GB2 generates the GG pdf with shape parameters $a>0$ and $c>0$, and scale parameter $p>0 .{ }^{7}$ Thus, condition (8) for the GG is given by

$$
m_{1, G G} \geq \frac{\Gamma\left(p+\frac{1}{c}\right)}{\Gamma(p)} \frac{\Gamma\left(p-\frac{1}{c}\right)}{\Gamma(p)} .
$$

This expression allows us to obtain results for other distributions nested within the GG. For instance, in the case of the two-parameter gamma pdf the condition for investors to allocate

\footnotetext{
${ }^{7}$ The GG family nests the gamma $(c=1)$, exponential $(p, c)=(1,1)$, Weibull $(p=1)$, log-normal $(p \rightarrow \infty)$ and Rayleigh $(p, c)=(1,2)$.
} 
all wealth in the risky asset can be solved solely in terms of the first and second moment, and is obtained as

$$
\begin{gathered}
1-E_{f}\left(r^{-1}\right)=1-a \frac{\Gamma\left(p-\frac{1}{1}\right)}{\Gamma(p)} \geq 0, \\
1-E_{f}\left(r^{-1}\right)=1-\frac{\Gamma(p+1)}{m_{1, g}} \frac{\Gamma(p-1)}{\Gamma(p)^{2}} \geq 0,
\end{gathered}
$$

which can be expressed in terms of central moments as

$$
\begin{aligned}
1-\frac{a}{p-1} & =1-\frac{\frac{m_{2, g}}{m_{1}, g}}{\frac{m_{1, g}^{2}}{m_{2, g}}-1} \geq 0 \\
m_{1, g} & \geq 1+\frac{m_{2, g}}{m_{1, g}} .
\end{aligned}
$$

For the case of the Weibull pdf the condition for the optimum is

$$
m_{1, W} \geq \Gamma\left(1+\frac{1}{c}\right) \Gamma\left(1-\frac{1}{c}\right) .
$$

Finally, we analyze the standard case of the log-normal pdf -assumed in Feldstein (1969), i.e., we assume that the logarithm of the risky asset (gross) return, $\ln (r)$, follows a Normal distribution with parameters $m$ and $v$

$$
\Phi(r ; m, v)=\frac{1}{r v \sqrt{2 \pi}} e^{-\frac{1}{2}\left(\frac{\ln (r)-m}{v}\right)^{2}}, 0<r<\infty .
$$

The mgf of this distribution is given by

$$
\mu_{t, L N}=E_{\Phi}\left[r^{t}\right]=\int r^{t} \Phi(r ; m, v) d r=e^{t m+\frac{1}{2} t^{2} v^{2}}, \forall t \in \mathbb{R} \text { or } \forall t \in \mathbb{C} .
$$

Thus, $\theta=1$ is optimum if the condition below holds

$$
\left.\xi_{\Phi}\left(u_{1}(\omega) ; \theta ; m, \nu\right)\right|_{\theta=1}=1-E_{\Phi}\left(r^{-1}\right) \geq 0
$$

which is expressed as

$$
\begin{aligned}
1 & \geq e^{-m+\frac{1}{2} v^{2}} \\
m & \geq \frac{1}{2} v^{2}
\end{aligned}
$$

Given that $\mu_{1, L N}=e^{m+\frac{1}{2} v^{2}}$ and $\mu_{2, L N}=e^{2 m+2 v^{2}}$ the condition above is: $2 \ln \mu_{1, L N}-$ $\frac{1}{2} \ln \mu_{2, L N} \geq \frac{1}{2} \ln \mu_{2, L N}-\ln \mu_{1, L N}$, or $3 \ln \mu_{1, L N} \geq \ln \mu_{2, L N}$. So we can write the condition for 
the optimum in terms of either the parameters (equation (21)), the first two raw moments (equation (22)) or the central moments (equation (23))

$$
\begin{aligned}
& \mu_{1, L N} \geq\left(1+\frac{\mu_{2, L N}-\mu_{1, L N}^{2}}{\mu_{1, L N}^{2}}\right), \\
& m_{1, L N} \geq 1+\frac{m_{2, L N}}{m_{1, L N}^{2}} .
\end{aligned}
$$

\section{The effect of higher-order moments in the investor's decision}

In this section we employ specific parameter values of the pdfs to illustrate the fact that more flexible pdfs allow the researcher to interpret portfolio decisions in terms of investors' broad risk profile rather than only in terms of risk aversion. The advantage of using the WGB2, and its nested pdfs, is that it allows to keep low(er) order moments constant while modifying high(er) order moments in order to examine the 'pure' effect that higher-order preferences play in portfolio selection. We note that previous studies in the literature typically limit their analysis to the impact of higher-order moments in portfolio weights, but they do not explicitly relate those weights to higher-order preferences. ${ }^{8}$

As the baseline for our comparison we employ the values in Feldstein, i.e., $m_{1, L N}=1.05$, a net return of $5 \%$. For this mean value, allocation of wealth in the risky asset will occur from condition (23) if the variance of the risky asset $m_{2, L N} \leq 0.055125$, or similarly, unless the standard deviation is about more than four times the expected net return, i.e., $m_{2, L N}^{1 / 2}>0.23479$. When the risky asset pdf has two-parameters, any two moments are sufficient to determine the optimal portfolio allocation. With a mean of 1.05 and a variance of 0.055125 , the third central moment of the log-normal takes the value of 0.008826 and the fourth central moment 0.011667 . The value of the variance appears practically relevant and hence Feldstein's original question as to why we do not appear to observe more investors not diversifying. In fact, if we consider an alternative two-parameter distribution such as the gamma (nested within the GG when $c=1$, Table 1) and still assume $m_{1, g}=1.05$, no-diversification is less likely since it occurs for a lower upper bound of the variance than in the log-normal (see Table 2, Panel A). ${ }^{9}$ Consequently, the Feldstein question could be even more salient.

\footnotetext{
${ }^{8} \mathrm{~A}$ paper that it does provide such a direct link is Níguez et al. (2016).

${ }^{9}$ Also note that for the Weibull no-diversification occurs when its variance is smaller than the variance of the gamma and log-normal.
} 
For the distributions with three, four and five parameters the conditions for agents to diversify depend on the higher moments but closed form analytic solutions cannot be obtained. In Table 2, Panel B, we show parameter values for two GG distributions that yield the same mean and variance as the lognormal presented above. However, for those GG pdfs investors would diversify rather than allocate all the wealth in the risky asset. ${ }^{10}$ This critical shift in the investors' optimal portfolio decision is due to downside risk aversion. The GG pdfs in this example imply more downside risk than the lognormal does, that is, they involve a transfer of probability weight leftward in the distribution preserving its mean and variance, making the individual worse off by such a change and therefore willing to diversify.

Employing an even more flexible pdf, the WGB2, where the optimal conditions are given by equation (9), we can demonstrate the effect of the fourth central moment. Using the same values of the mean, variance, and skewness that imply agents are no-diversifiers when the distribution is log normal, we find parameter values for the WGB2, namely, $(p, c, q, b, k)=(4.92879,2.80226,6.5,1.1025791,0.7)$, such that the individual diversifies rather than allocates all wealth in the risky asset. This is because the value of the fourth moment for the WGB2 is higher than that for the $\log$ normal $\left(m_{4, W G B 2}=\right.$ $\left.0.01224027, m_{4, L N}=0.01162909\right) .{ }^{11}$ Figure 1 illustrates the differences of the pdfs considered in this latter example.

\section{[Insert Table 2 and Figure 1]}

\section{Conclusions}

In this paper we showed that flexible pdfs that are typically employed to capture stylized features of asset returns such as skewness and leptokurtosis are a useful tool to assess the impact that higher-order moments have on the optimal behavior of investors. In particular, the five-parameter WGB2 allows the identification of pure effects that higher-order risk preferences such as prudence and temperance have on portfolio choice. This is the case because low(er) order moments can be held equal across alternative pdfs while high(er) moments can be modified to reflect agents' preferences.

\footnotetext{
${ }^{10}$ The EU for those two GG is lower than the EU of the log-normal. In particular, $E U_{L N}=0.0243951$, (hereafter $E U_{f}$ denotes EU under density $f$ ) and for the two GG in Table 2 Panel B, $E U_{G G}=0.0227413$ and $E U_{G G}=0.0237374$.

${ }^{11}$ An application of the no-diversification conditions derived in this section, for the S\&P500 index data in Robert Shiller's webpage, is available upon request. We find that the fitted distributions that are most flexible (GB2, GG) display closer moments to those of the data and present better fit according to information criteria.
} 


\section{References}

[1] Brandt, M. 2010. Portfolio Choice Problems, in Handbook of Financial Econometrics Volume 1: Tools and Techniques, edited by Y. Ait-Sahalia and L. P. Hansen. NorthHolland.

[2] Brunnermeier, M., and Nagel, S. 2008. Do Wealth Fluctuations Generate Time-Varying Risk Aversion? Micro-Evidence on Individuals' Asset Allocation. American Economic Review 98, 713-736.

[3] Eeckhoudt, L., and Schlesinger, H. 2006. Putting Risk in its Proper Place. American Economic Review 96, 280-289.

[4] Feldstein, M. S. 1969. Mean-Variance Analysis in the Theory of Liquidity Preference and Portfolio Selection. Review of Economic Studies 36, 5-12.

[5] Gandelman, N. and Hernandez-Murillo, R. 2014. Risk Aversion at the Country Level. Federal Reserve Bank of Saint Louis Working Paper 2014-005B.

[6] Guidolin, M., and Timmermann, A. 2008. International Asset Allocation under Regime Switching, Skew, and Kurtosis Preferences. Review of Financial Studies 21, 889-935.

[7] Hartley, R., Lanot, G., and Walker, I. 2014. Who Really Wants to Be a Millionaire? Estimates of Risk Aversion from a Game Show. Journal of Applied Econometrics 29, 861-879.

[8] Kimball, M. S. 1990. Precautionary Saving in the Small and in the Large. Econometrica $58,53-73$.

[9] McDonald, J. B. 1984. Some Generalized Functions for the Size Distribution of Income. Econometrica 52, 647-663.

[10] Menezes, C., Geiss, C., and Tressler, J. 1980. Increasing Downside Risk. American Economic Review 70, 921-32.

[11] Ñ́guez, T. M., Paya, I., and Peel, D. A. 2016. Pure Higher Order Effects in the Portfolio Choice Model. Finance Research Letters 19, 255-260.

[12] Ye, Y., Oluyede, B. O., and Pararai, M. 2012. Weighted Generalized Beta Distribution of the Second Kind and Related Distributions. Journal of Statistical and Econometric Methods 1, 13-31. 


\section{Figures}

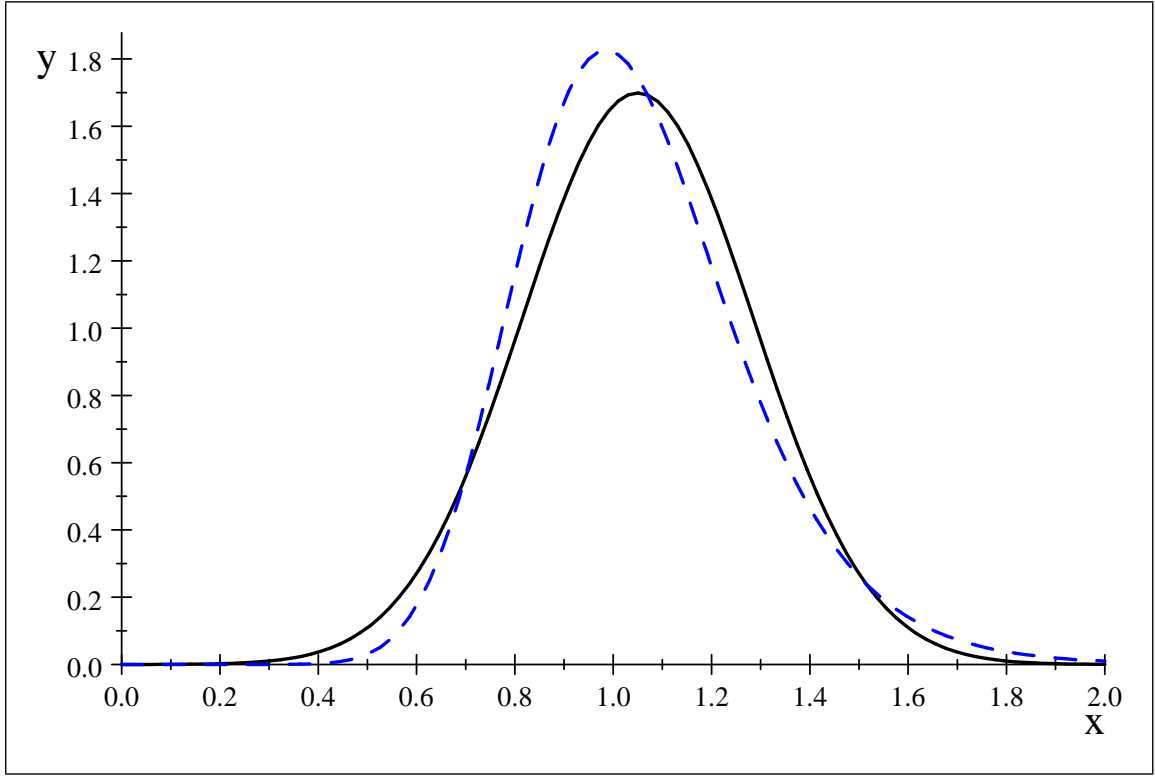

FIGURE 1. Pdfs of the log normal (black solid line) and WGB2 (blue dash line). 


\section{Tables}

\section{TABLE 1}

Density and moment generating functions of the generalized distributions

\begin{tabular}{|c|c|c|}
\hline & $p d f$ & $m g f=E\left[r^{t}\right]$ \\
\hline$W G B 2(r ; k, c, b, p, q)$ & $\frac{c r^{c p+k-1} \Gamma(p+q)}{b^{c p+k} \Gamma\left(p+\frac{k}{c}\right) \Gamma\left(q-\frac{k}{c}\right)\left(1+\frac{r^{c}}{b^{c}}\right)^{p+q}}$ & $\frac{b^{t} \Gamma\left(p+\frac{k}{c}+\frac{t}{c}\right) \Gamma\left(q-\frac{k}{c}-\frac{t}{c}\right)}{\Gamma\left(p+\frac{k}{c}\right) \Gamma\left(q-\frac{k}{c}\right)}$ \\
\hline$G B 2(r ; c, b, p, q)$ & $\frac{c r^{c p-1} \Gamma(p+q)}{b^{c p} \Gamma(p) \Gamma(q)\left(1+\frac{r^{c}}{b^{c}}\right)^{p+q}}$ & $\frac{b^{t} \Gamma\left(p+\frac{t}{c}\right) \Gamma\left(q-\frac{t}{c}\right)}{\Gamma(p) \Gamma(q)}$ \\
\hline$G G(r ; a, p, c)$ & $\frac{c a^{c p} r^{c p-1} e^{-(a r)^{c}}}{\Gamma(p)}$ & $\frac{1}{a^{t}} \frac{\Gamma\left(p+\frac{t}{c}\right)}{\Gamma(p)}$ \\
\hline
\end{tabular}

Notes: Pdfs and mgfs of WGB2, GB2 and GG distributions. $\Gamma(p)=\int_{0}^{\infty} e^{-r} r^{p-1} d r$ denotes the gamma function. Parameter $k$ controls the shape and skewness of the WGB2 density, which nests the GB2 when $k=0$, which, in turns, nests the GG when $b=a^{-1} q^{1 / c}$ as $q \rightarrow \infty$. 


\section{TABLE 2}

GB2-class of densities and diversification: Range of moments and $p d f$ specification

\begin{tabular}{|c|c|c|c|c|c|}
\hline & GB2 & GG & gamma & Weibull & log-normal \\
\hline \multicolumn{6}{|c|}{ Panel A. Maximum $m_{2}$ for which ND holds within a class of pdf } \\
\hline$m_{2}^{*}$ & 0.0581 & 0.055125 & 0.05250 & 0.043580 & 0.055125 \\
\hline$m_{3}$ & 0.0141 & 0.008826 & 0.00525 & -0.00323 & 0.008826 \\
\hline ND & Yes & Yes & Yes & Yes & Yes \\
\hline \multicolumn{6}{|c|}{ Panel B. Examples of GG distributions with same $\left(m_{1}, m_{2}\right)$ as log-normal in Panel $A$} \\
\hline \multicolumn{6}{|c|}{ GG that nests log-normal: $(c, p, a)=(2,5.11592,2.1022)$} \\
\hline$m_{2}$ & & 0.055125 & & & 0.055125 \\
\hline$m_{3}$ & & 0.003034 & & & 0.008826 \\
\hline ND & & No & & & Yes \\
\hline \multicolumn{6}{|c|}{ GG that nests log-normal: $(c, p, a)=(0.81694,30,61.503)$} \\
\hline$m_{2}$ & & 0.055125 & & & 0.055125 \\
\hline$m_{3}$ & & 0.006324 & & & 0.008826 \\
\hline ND & & No & & & Yes \\
\hline
\end{tabular}

Panel C. Example of two GB2 pdfs with same $\left(m_{1}, s k\right)$ and ND holds for higher $m_{2}$

\begin{tabular}{lcl} 
& \multicolumn{2}{c}{ GB2 with $\left(m_{1}, s k\right)=(1.05,2.194406)$} \\
$p$ & 1.77451 & 22 \\
$c$ & 7.574 & 4.5 \\
$q$ & 0.85 & 1.5 \\
$b$ & 0.88208 & 0.52162 \\
$m_{2}$ & 0.06819 & 0.06836 \\
ND & No & Yes \\
\hline
\end{tabular}

Notes: Summary of the condition under which the investor would optimally allocate all wealth in the risky asset (ND) for the examples of the GB2 pdfs presented in this section. For all cases $m_{1}=1.05$ and $m_{2}^{*}$ denotes the maximum variance so that no-diversification (ND) condition holds.

\section{Word count: 2,042}

